Bull. Korean Math. Soc. 50 (2013), No. 5, pp. 1651-1657

http://dx.doi.org/10.4134/BKMS.2013.50.5.1651

\title{
DERIVATIONS WITH ANNIHILATOR CONDITIONS IN PRIME RINGS
}

\author{
Basudeb Dhara, Sukhendu Kar, And Sachhidananda Mondal \\ AbSTRACt. Let $R$ be a prime ring, $I$ a nonzero ideal of $R, d$ a derivation \\ of $R, m(\geq 1), n(\geq 1)$ two fixed integers and $a \in R$. (i) If $a((d(x) y+$ \\ $\left.x d(y)+d(y) x+y d(x))^{n}-(x y+y x)\right)^{m}=0$ for all $x, y \in I$, then either \\ $a=0$ or $R$ is commutative; (ii) If $\operatorname{char}(R) \neq 2$ and $a((d(x) y+x d(y)+$ \\ $\left.d(y) x+y d(x))^{n}-(x y+y x)\right) \in Z(R)$ for all $x, y \in I$, then either $a=0$ or \\ $R$ is commutative.
}

\section{Introduction}

Throughout this paper $R$ always denotes a prime ring with center $Z(R)$, extended centroid $C$ and $Q$ its two-sided Martindale quotient ring. Recall that a ring $R$ is said to be prime, if for any $a, b \in R, a R b=(0)$ implies either $a=0$ or $b=0$ and it is semiprime if for any $a \in R, a R a=(0)$ implies $a=0$. For any $x, y \in R$, the Lie commutator of $x, y$ is denoted by $[x, y]$ and defined by $[x, y]=x y-y x$ and the anti-commutator is denoted by $x \circ y$ and is defined by $x \circ y=x y+y x$. By $d$ we mean a derivation of $R$. A derivation $d$ is inner if there exists $b \in R$ such that $d(x)=[b, x]$ holds for all $x \in R$.

A well known theorem of Posner [15] states that if $R$ is prime and the commutator $[d(x), x] \in Z(R)$ for all $x \in R$, then either $d=0$ or $R$ is commutative. This result of Posner was generalized in many directions by several authors and they studied the relationship between the structure of prime or semiprime ring and the behaviour of additive maps satisfying various conditions. Some authors have studied the derivations with annihilator conditions in prime and semiprime rings (see [3], [4], [6], [7], [8], [9]; where further references can be found).

In [2], Ashraf and Rehman proved that if $R$ is a prime ring, $I$ is a nonzero ideal of $R$ and $d$ is a derivation of $R$ such that $d(x) y+x d(y)+d(y) x+y d(x)=$ $x y+y x$ for all $x, y \in I$, then $R$ is commutative. Recently, in [1]; Argac and Inceboz generalized the above result as follows:

Received October 19, 2012.

2010 Mathematics Subject Classification. 16W25, 16R50, 16N60.

Key words and phrases. prime ring, derivation, extended centroid. 
Let $R$ be a prime ring, $I$ a nonzero ideal of $R, d$ a derivation of $R$ and $n$ a fixed positive integer. (i) If $(d(x) y+x d(y)+d(y) x+y d(x))^{n}=x y+y x$ for all $x, y \in I$, then $R$ is commutative. (ii) If $\operatorname{char}(R) \neq 2$ and $(d(x) y+x d(y)+$ $d(y) x+y d(x))^{n}-x y+y x$ is central for all $x, y \in I$, then $R$ is commutative.

In this present paper we study this situation with annihilator condition in more generalized way. We mainly prove the following results:

Let $R$ be a prime ring, $I$ a nonzero ideal of $R, d$ a derivation of $R, m(\geq$ $1), n(\geq 1)$ two fixed integers and $a \in R$. (i) If $a((d(x) y+x d(y)+d(y) x+$ $\left.y d(x))^{n}-(x y+y x)\right)^{m}=0$ for all $x, y \in I$, then either $a=0$ or $R$ is commutative; (ii) If $\operatorname{char}(R) \neq 2$ and $a\left((d(x) y+x d(y)+d(y) x+y d(x))^{n}-(x y+y x)\right) \in Z(R)$ for all $x, y \in I$, then either $a=0$ or $R$ is commutative.

\section{Main results}

We first begin with the following lemma:

Lemma 2.1. Let $R$ be a prime ring, $b \in Q$ and $m, n(\geq 1)$ fixed integers. If $0 \neq a \in R$ such that $a\left([b, x y+y x]^{n}-(x y+y x)\right)^{m}=0$ for all $x, y \in R$, then either $b \in C$ or $R$ satisfies a nontrivial generalized polynomial identity (GPI).

Proof. Let $T=R *_{C} C\{x, y\}$ be the free product of $R$ and $C\{x, y\}$, where $C\{x, y\}$ is the free $C$-algebra in noncommuting indeterminates $x$ and $y$. Suppose that $R$ does not satisfy any nontrivial GPI. Then, we find that $a([b, x y+$ $\left.y x]^{n}-(x y+y x)\right)^{m}$ is the zero element in the free product $T$, that is

$$
a\left([b, x y+y x]^{n}-(x y+y x)\right)^{m}=0
$$

in $T$. This can be re-written as

$$
\begin{array}{r}
a\left([b, x y+y x]^{n}-(x y+y x)\right)^{m-1} \\
\cdot\left([b, x y+y x]^{n-1}(b(x y+y x)-(x y+y x) b)-(x y+y x)\right)=0 .
\end{array}
$$

We assume that $b \notin C$. Then 1 and $b$ are linearly independent over $C$. Since (1) is a trivial GPI, (1) implies that

$$
a\left([b, x y+y x]^{n}-(x y+y x)\right)^{m-1}\left([b, x y+y x]^{n-1}(x y+y x) b\right)=0
$$

and so

$$
a\left([b, x y+y x]^{n}-(x y+y x)\right)^{m-1}((x y+y x) b)^{n}=0
$$

in $T$. Continuing the same process, it reduces to $a((x y+y x) b)^{m n}=0 \in T$, implying $b=0$, a contradiction. Hence we conclude that $b \in C$, as desired.

Theorem 2.2. Let $R$ be a prime ring, $I$ a non zero ideal of $R, d$ a derivation of $R, m(\geq 1), n(\geq 1)$ two fixed integers. If $a \in R$ such that $a((d(x) y+x d(y)+$ $\left.d(y) x+y d(x))^{n}-(x y+y x)\right)^{m}=0$ for all $x, y \in I$, then either $a=0$ or $R$ is commutative. 
Proof. Let $a \neq 0$. By our assumption

$$
a\left((d(x) y+x d(y)+d(y) x+y d(x))^{n}-(x y+y x)\right)^{m}=0
$$

for all $x, y \in I$. Let $d=0$. Then from (4) we get $(-1)^{m} a(x y+y x)^{m}=0$, that is $a(x y+y x)^{m}=0$ for all $x, y \in I$. This is a generalized polynomial identity (GPI) for $I$. By Chuang [5, Theorem 2], this GPI is also satisfied by $Q$ and hence by $R$. Let $w=(x y+y x)^{m}$. Then $a w=0$. Thus we can write $a(p w q a+w q a p)^{m}=0$ for all $p, q \in R$. Since $a w=0$, it reduces to $a(p w q a)^{m}=0$. This can be written as $(\text { wqap })^{m+1}=0$ for all $p, q \in R$. By Levitzki's lemma [11, Lemma 1.1], $w q a=0$ for all $q \in R$. Since $R$ is prime, either $a=0$ or $w=0$. Since $a \neq 0, w=(x y+y x)^{m}=0$ for all $x, y \in R$. This is a polynomial identity (PI) for $R$. So by Lemma 1 in [13], there exists a suitable field $F$ such that $R \subseteq M_{k}(F)$, the ring of all $k \times k$ matrices over $F$, and moreover $M_{k}(F)$ satisfies the same polynomial identity as $R$ satisfies. Let $k \geq 2$. In this case, if we take $x=e_{12}, y=e_{21}$, then we find a contradiction that $0=(x y+y x)^{m}=e_{11}+e_{22}$. Hence $k=1$ and so $R$ is commutative.

Next we assume that $d \neq 0$. Then $a\left((d(x) y+x d(y)+d(y) x+y d(x))^{n}-\right.$ $(x y+y x))^{m}=0$ is a differential identity for $I$. If $d$ is not $Q$-inner, then by Kharchenko's theorem [12], I satisfies the identity $a\left((r y+x s+s x+y r)^{n}-\right.$ $(x y+y x))^{m}=0$. In particular for $r=s=0$, we find that $a(x y+y x)^{m}=0$ for all $x, y \in I$. Then $R$ is commutative as above. Now let $d$ be $Q$-inner, i.e., $d(x)=[b, x]$ for all $x \in R$ and for some $b \in Q$. Since $d \neq 0, b \notin C$. Then (4) becomes

$$
a\left(([b, x] y+x[b, y]+[b, y] x+y[b, x])^{n}-(x y+y x)\right)^{m}=0
$$

for all $x, y \in I$. By Chuang [5, Theorem 2], this GPI is also satisfied by $Q$, that is,

(6) $\quad f(x, y)=a\left(([b, x] y+x[b, y]+[b, y] x+y[b, x])^{n}-(x y+y x)\right)^{m}=0$

for all $x, y \in Q$. In case the center $C$ of $Q$ is infinite, we have $f(x, y)=0$ for all $x, y \in Q \bigotimes_{C} \bar{C}$, where $\bar{C}$ is the algebraic closure of $C$. Since both $Q$ and $Q \otimes_{C} \bar{C}$ are prime and centrally closed [10, Theorems 2.5 and 3.5], we may replace $R$ by $Q$ or $Q \otimes_{C} \bar{C}$ according as $C$ is finite or infinite. Thus we may assume that $R$ is centrally closed over $C$ (i.e., $R C=R$ ), which is either finite or algebraically closed and $f(x, y)=0$ for all $x, y \in R$. By Lemma 2.1, it follows that $f(x, y)=0$ is a nontrivial GPI for $R$. Then by Martindale's theorem [14], $R C=R$ is a primitive ring with nonzero socle and $C$ is the associated division ring. Hence $R$ is isomorphic to a dense ring of linear transformations of a vector space $V$ over $C$. If $V$ is finite dimensional over $C$, then the density of $R$ on $V$ implies that $R \cong M_{k}(C)$, where $k=\operatorname{dim}_{C} V$.

Suppose that $\operatorname{dim}_{C} V \geq 3$.

We shall show that for any $v \in V, v$ and $b v$ are linearly $C$-dependent. Suppose that $v$ and $b v$ are linearly $C$-independent for some $v \in V$. Since $\operatorname{dim}_{C} V \geq 3$, there exists $w \in V$ such that $v, b v, w$ are linearly $C$-independent. 
By density there exist $x, y \in R$ such that

$$
\begin{aligned}
& x v=0, x b v=w, x w=0 ; \\
& y v=0, y b v=0, y w=v .
\end{aligned}
$$

Then $0=a\left(([b, x] y+x[b, y]+[b, y] x+y[b, x])^{n}-(x y+y x)\right)^{m} v=(-1)^{m n} a v$. This implies that if $a v \neq 0$, then by contrary $v$ and $b v$ are linearly $C$-dependent. Now suppose that $a v=0$. Since $a \neq 0$, there exists $w \in V$ such that $a w \neq 0$ and then $a(v+w)=a w \neq 0$. By the previous argument, we find that $w, b w$ are linearly $C$-dependent and also so are $v+w, b(v+w)$. Thus there exists $\alpha, \beta \in C$ such that $b w=w \alpha$ and $b(v+w)=(v+w) \beta$. Moreover, $v$ and $w$ are linearly $C$-independent and so by density there exist $x, y \in R$ such that

$$
\begin{aligned}
& x w=v, x v=0 ; \\
& y w=0, y v=-w(\alpha-\beta) .
\end{aligned}
$$

Then we obtain that $0=a\left(([b, x] y+x[b, y]+[b, y] x+y[b, x])^{n}-(x y+y x)\right)^{m} w=$ $a w(\alpha-\beta)^{m}$. Since $a w \neq 0, \alpha=\beta$ and so $b v=v \alpha$, a contradiction. Hence we conclude that for any $v \in V, v$ and $b v$ are linearly $C$-dependent, that is $b v=v \alpha_{v}$ for some $\alpha_{v} \in C$. It is very easy to prove that $\alpha_{v}$ is independent of the choice of $v \in V$. Thus we can write $b v=v \alpha$ for all $v \in V$ and $\alpha \in C$ fixed. Now let $r \in R, v \in V$. Since $b v=v \alpha$, we have

$$
[b, r] v=(b r) v-(r b) v=b(r v)-r(b v)=(r v) \alpha-r(v \alpha)=0 .
$$

Thus $[b, r] v=0$ for all $v \in V$, i.e., $[b, r] V=0$. Since $[b, r]$ acts faithfully as a linear transformation on the vector space $V,[b, r]=0$ for all $r \in R$. Therefore, $b \in Z(R)$ which implies $d=0$, a contradiction.

If $\operatorname{dim}_{C} V=1$, then $R$ is commutative and we are done in this case.

Now suppose $\operatorname{dim}_{C} V=2$. Then $R \cong M_{2}(C)$. Then $M_{2}(C)$ satisfies the identity $a\left(([b, x] y+x[b, y]+[b, y] x+y[b, x])^{n}-(x y+y x)\right)^{m}=0$. But by choosing $x=e_{12}, y=e_{21}$, we arrive at a contradiction that $0=(-1)^{m} a\left(e_{11}+e_{22}\right)=$ $(-1)^{m} a I_{2}=(-1)^{m} a$.

This completes the proof of the theorem.

Lemma 2.3. Let $R=M_{k}(F), k \geq 2$ be the ring of all $k \times k$ matrices over $a$ field $F$ and $a \in R$. If for some $b \in R, a\left(([b, x] y+x[b, y]+[b, y] x+y[b, x])^{n}-\right.$ $(x y+y x)) \in Z(R)$ for all $x, y \in R$, where $n(\geq 1)$ is a fixed integer, then $a=0$.

Proof. Let $a=\left(a_{i j}\right)_{k \times k}$ and $b=\left(b_{i j}\right)_{k \times k}$. If for all $x, y \in M_{k}(F)$,

$$
a\left(([b, x] y+x[b, y]+[b, y] x+y[b, x])^{n}-(x y+y x)\right)=0
$$

then by Theorem 2.2, we find that $a=0$, as desired. So, let for some $x, y \in$ $M_{k}(F)$,

$$
a\left(([b, x] y+x[b, y]+[b, y] x+y[b, x])^{n}-(x y+y x)\right) \neq 0
$$


Then $a$ is invertible. Thus for each $x, y \in M_{k}(F)$,

$$
\left(([b, x] y+x[b, y]+[b, y] x+y[b, x])^{n}-(x y+y x)\right) \in F \cdot a^{-1} .
$$

Therefore, for each $x, y \in M_{k}(F)$, either

$$
\left(([b, x] y+x[b, y]+[b, y] x+y[b, x])^{n}-(x y+y x)\right)
$$

is zero or invertible in $M_{k}(F)$.

Now we consider the following two cases:

Case-1: $k \geq 3$.

We choose $x=e_{11}, y=e_{12}$. Then $x y+y x=e_{12}$ and $([b, x] y+x[b, y]+$ $[b, y] x+y[b, x])^{n}-(x y+y x)=\left[b, e_{12}\right]^{n}-e_{12}$. Since rank of $\left[b, e_{12}\right]^{n}-e_{12}$ is $\leq 2$, it can not be invertible and so $\left[b, e_{12}\right]^{n}-e_{12}=0$. Left multiplying by $e_{12}$, we get

$$
0=e_{12}\left(\left[b, e_{12}\right]^{n}-e_{12}\right)=e_{12}\left(b e_{12}\right)^{n}=b_{21}^{n} e_{12}
$$

implying $b_{21}=0$. Thus for any $i \neq j, b_{i j}=0$ implying $b$ is diagonal. Let $b=\sum_{i=1}^{k} b_{i i} e_{i i}$. For any $F$-automorphism $\theta$ of $R, b^{\theta}$ enjoys the same property as $b$ does, namely, $\left(\left[b^{\theta}, x\right] y+x\left[b^{\theta}, y\right]+\left[b^{\theta}, y\right] x+y\left[b^{\theta}, x\right]\right)^{n}-(x y+y x)$ is zero or invertible for every $x, y \in R$. Hence $b^{\theta}$ must be diagonal. For each $j \neq 1$, we have $\left(1+e_{1 j}\right) b\left(1-e_{1 j}\right)=\sum_{i=1}^{k} b_{i i} e_{i i}+\left(b_{j j}-b_{11}\right) e_{1 j}$ diagonal. Therefore, $b_{j j}=b_{11}$ and so $b \in F \cdot I_{k}$. Then our identity reduces to $(x y+y x) \in F \cdot a^{-1}$ for all $x, y \in R$. But by choosing $x=e_{11}, y=e_{12}$, we find that $(x y+y x)=e_{12}$ which is neither zero nor invertible in $R$, a contradiction.

Case- $2: k=2$.

We choose $x=e_{12}, y=e_{21}$. Then we have $x y+y x=e_{11}+e_{22}=I_{2}$ and $([b, x] y+x[b, y]+[b, y] x+y[b, x])^{n}-(x y+y x)=\left[b, I_{2}\right]^{n}-I_{2}=-I_{2} \in F \cdot a^{-1}$. This gives $a \in F \cdot I_{2}$. Thus our assumption becomes $([b, x] y+x[b, y]+[b, y] x+$ $y[b, x])^{n}-(x y+y x) \in F \cdot I_{2}$ for all $x, y \in R$. If $n$ is even then $([b, x] y+x[b, y]+$ $[b, y] x+y[b, x])^{n}=[b, x y+y x]^{n} \in F \cdot I_{2}$ and hence $(x y+y x) \in F \cdot I_{2}$ for all $x, y \in R$. This leads to a contradiction, since $(x y+y x)=e_{11} e_{12}+e_{12} e_{11}=$ $e_{12} \notin F \cdot I_{2}$. Thus we assume that $n$ is an odd integer. In this case, we choose $x=e_{11}, y=e_{12}$ and then obtain $([b, x] y+x[b, y]+[b, y] x+y[b, x])^{n}-(x y+y x)=$ $\left[b, e_{12}\right]^{n}-e_{12} \in F \cdot I_{2}$. Commuting both sides with $e_{12}$, we have $0=\left[\left[b, e_{12}\right]^{n}-\right.$ $\left.e_{12}, e_{12}\right]=\left[b, e_{12}\right]^{n} e_{12}-e_{12}\left[b, e_{12}\right]^{n}=(-1)^{n} b_{21}^{n} e_{12}-b_{21}^{n} e_{12}=-2 b_{21}^{n} e_{12}$. Since char $(R) \neq 2$, we get $b_{21}=0$. Thus for any $i \neq j, b_{i j}=0$ that is, $b$ is diagonal. Then by same argument as above we conclude that $b$ is central, that is $b \in F \cdot I_{2}$. Thus we have $(x y+y x) \in F \cdot I_{2}$ for all $x, y \in R$. But this case leads to a contradiction, since $(x y+y x)=e_{11} e_{12}+e_{12} e_{11}=e_{12} \notin F \cdot I_{2}$.

Theorem 2.4. Let $R$ be a prime ring with char $(R) \neq 2, I$ a nonzero ideal of $R, d$ a derivation of $R$. If $a \in R$ such that $a\left((d(x) y+x d(y)+d(y) x+y d(x))^{n}-\right.$ $(x y+y x)) \in Z(R)$ for all $x, y \in I$, where $n \geq 1$ is a fixed integer, then either $a=0$ or $R$ is commutative. 
Proof. By our assumption

$$
a\left((d(x) y+x d(y)+d(y) x+y d(x))^{n}-(x y+y x)\right) \in Z(R)
$$

for all $x, y \in I$. If $a\left((d(x) y+x d(y)+d(y) x+y d(x))^{n}-(x y+y x)\right)=0$ for all $x, y \in I$, then by Theorem 2.2, we get our conclusion. So let there exist some $x, y \in I$ such that $0 \neq a\left((d(x) y+x d(y)+d(y) x+y d(x))^{n}-(x y+y x)\right) \in I \cap Z(R)$. Thus $I \cap Z(R) \neq 0$. Let $K$ be a nonzero two-sided ideal of $R_{Z}$, the ring of the central quotients of $R$. Since $K \cap R$ is an ideal of $R,(K \cap R) \cap Z(R) \neq 0$. Hence $K$ contains an invertible element in $R_{Z}$, and so $R_{Z}$ is a simple ring with 1.

By the hypothesis for any $x, y \in I$ and $r \in R$, we have

$$
\left[a\left((d(x) y+x d(y)+d(y) x+y d(x))^{n}-(x y+y x)\right), r\right]=0 .
$$

If $d$ is not $Q$-inner then by Kharchenko's theorem [12],

$$
\left[a\left((s y+x t+t x+y s)^{n}-(x y+y x)\right), r\right]=0
$$

for all $x, y, s, t \in I$ and $r \in R$. By Chuang [5], this GPI of (9) is also satisfied by $Q$ and hence by $R$ too. By localizing $R$ at $Z(R)$, it follows that $[a((s y+$ $\left.\left.x t+t x+y s)^{n}-(x y+y x)\right), r\right]$ is also an identity of $R_{Z}$. Since $R$ and $R_{Z}$ satisfy the same polynomial identity, in order to prove that $R$ is commutative, we may assume that $R$ is simple ring with 1 . Thus $R$ satisfies the identity (9). If $R$ is commutative we are done. So, let $R$ be noncommutative. Then there exists $b \in R$ such that $b \notin Z(R)$. Now putting $s=[b, x]$ and $t=[b, y]$, we obtain that $R$ satisfies

$$
\left[a\left(([b, x] y+x[b, y]+[b, y] x+y[b, x])^{n}-(x y+y x)\right), r\right]=0 .
$$

By Martindale's theorem [14], $R$ is a primitive ring with minimal right ideal, whose commuting ring $D$ is a division ring which is finite dimensional over $Z(R)$. However, since $R$ is simple with $1, R$ must be Artinian. Hence $R=D_{k^{\prime}}$, the $k^{\prime} \times k^{\prime}$ matrices over $D$, for some $k^{\prime} \geq 2$. Again, by [13, Lemma 2], it follows that there exists a field $F$ such that $R \subseteq M_{k}(F)$, the ring of $k \times k$ matrices over the field $F$, and $M_{k}(F)$ satisfies

$$
\left[a\left(([b, x] y+x[b, y]+[b, y] x+y[b, x])^{n}-(x y+y x)\right), r\right]=0,
$$

that is,

$$
a\left(([b, x] y+x[b, y]+[b, y] x+y[b, x])^{n}-(x y+y x)\right) \in Z\left(M_{k}(F)\right) .
$$

Then by Lemma 2.3, we conclude that $a=0$.

By the same argument as above, we can draw the same conclusion in case $d$ is a $Q$-inner derivation that is $d(x)=[b, x]$ for all $x \in R$ and for some $b \in Q$. 


\section{References}

[1] N. Argac and H. G. Inceboz, Derivations of prime and semiprime rings, J. Korean Math. Soc. 46 (2009), no. 5, 997-1005.

[2] M. Ashraf and N. Rehman, On commutativity of rings with derivations, Results Math. 42 (2002), no. 1-2, 3-8.

[3] L. Carini, V. De Filippis, and B. Dhara, Annihilators on co-commutators with generalized derivations on Lie ideals, Publ. Math. Debrecen 76 (2010), no. 3-4, 395-409.

[4] C. M. Chang and T. K. Lee, Annihilators of power values of derivations in prime rings, Comm. Algebra 26 (1998), no. 7, 2091-2113.

[5] C. L. Chuang, GPI's having coefficients in Utumi quotient rings, Proc. Amer. Math. Soc. 103 (1988), no. 3, 723-728.

[6] V. De Filippis, Lie ideals and annihilator conditions on power values of commutators with derivation, Indian J. Pure Appl. Math. 32 (2001), no. 5, 649-656.

[7] B. Dhara, Power values of derivations with annihilator conditions on Lie ideals in prime rings, Comm. Algebra 37 (2009), no. 6, 2159-2167.

[8] - Annihilator condition on power values of derivations, Indian J. Pure Appl. Math. 42 (2011), no. 5, 357-369.

[9] - Left annihilators of power values of commutators with generalized derivations, Georgian Math. J. 19 (2012), no. 3, 441-448.

[10] T. S. Erickson, W. S. Martindale III, and J. M. Osborn, Prime nonassociative algebras, Pacific J. Math. 60 (1975), no. 1, 49-63.

[11] I. N. Herstein, Topics in Ring Theory, Univ. of Chicago Press, Chicago, 1969.

[12] V. K. Kharchenko, Differantial identities of prime rings, Algebra i Logika 17 (1978), no. 2, 220-238.

[13] C. Lanski, An Engel condition with derivation, Proc. Amer. Math. Soc. 118 (1993), no. $3,731-734$

[14] W. S. Martindale III, Prime rings satisfying a generalized polynomial identity, J. Algebra 12 (1969), 576-584.

[15] E. C. Posner, Derivations in prime rings, Proc. Amer. Math. Soc. 8 (1957), 1093-1100.

Basudeb Dhara

Department of Mathematics

Belda College, Belda

PAschim Medinipur

721424(W.B.), INDIA

E-mail address: basu_dhara@yahoo.com

SUKHENDU KAR

Department of Mathematics

JADAVPUR UNIVERSITY

KOLKATA-700032, INDIA

E-mail address: karsukhendu@yahoo.co.in

SACHHIDANANDA MONDAL

DEPARTMENT OF MATHEMATICS

JADAVPUR UNIVERSITY

KOLKATA-700032, INDIA

E-mail address: sachhidananda.mondal@gmail.com 